Commun. Math. Phys. 150, 561-583 (1992)

Communications in

Mathematical

Physics

(C) Springer-Verlag 1992

\title{
Global Existence of Solutions of the Spherically Symmetric Vlasov-Einstein System with Small Initial Data
}

\author{
G. Rein ${ }^{1}$ and A.D. Rendall ${ }^{2}$ \\ ${ }^{1}$ Mathematisches Institut der Universität München, Theresienstr. 39, W-8000 München 2, \\ Germany \\ ${ }^{2}$ Max-Planck-Institut für Astrophysik, Karl-Schwarzschild-Str. 1, W-8046 Garching bei \\ München, Germany
}

Received March 25, 1992

\begin{abstract}
We show that global asymptotically flat singularity-free solutions of the spherically symmetric Vlasov-Einstein system exist for all initial data which are sufficiently small in an appropriate sense. At the same time detailed information is obtained concerning the asymptotic behaviour of these solutions. A key element of the proof which is also of intrinsic interest is a local existence theorem with a continuation criterion which says that a solution cannot cease to exist as long as the maximum momentum in the support of the distribution function remains bounded. These results are contrasted with known theorems on spherically symmetric dust solutions.
\end{abstract}

\section{Introduction}

The Vlasov-Einstein system describes a self-gravitating collisionless gas within the framework of general relativity. The only available theorem on the initial value problem for this system is a local in time existence result due to Choquet-Bruhat [3]. There are two reasons why it is of interest to study this initial value problem. In recent years there has been considerable progress in understanding the closely related Vlasov-Poisson and Vlasov-Maxwell systems and similar equations. In particular, it has been shown by Pfaffelmoser [19] (see also [17, 23]) that the Vlasov-Poisson system, which is the non-relativistic analogue of the VlasovEinstein system, has global classical solutions for general initial data. In the case of the Vlasov-Maxwell system the corresponding question is still open but a number of partial results have been obtained. (For details see [20].) Since the VlasovEinstein system, which up to now has been studied very little, has deep relations to these other equations, one motivation for studying it is to obtain a better understanding of the initial value problem for a whole class of differential equations which are of intrinsic mathematical interest and have varied physical applications. The other motivation is that the Vlasov equation provides a matter model for general relativity which seems particularly suitable for the study of the long-time behaviour of matter in gravitational fields. 
To see the special role of the collisionless gas among matter models which can be used in general relativity, note that the global existence theorem for the Vlasov-Poisson system mentioned above implies that it is reasonable to expect global existence of classical solutions of the equations describing this kind of matter in rather general situations. This contrasts with other kinds of matter where it is known that singularities occur even for small initial data. Notable examples are perfect fluids [24] and elastic solids [16]. Of course it is possible to concentrate on the case where spacetime is empty or only contains radiation, and very important results have been obtained in this direction [5-11]. If, however, one wishes to describe "normal matter" (i.e. matter which from a physical point of view ultimately consists of massive particles) then the only known reasonable candidate for a type of matter which does not form singularities of its own accord is the collisionless gas. The importance of such singularities is that they may prevent the investigation of the spacetime singularities which are essentially gravitational in nature and which are of central interest in general relativity. (For a more detailed discussion of this point see [22].) Support for these ideas, which are based on plausible analogies, is provided by the main theorem of this paper (Theorem 4.1) which shows that global singularity-free solutions of the Vlasov-Einstein system exist in one particular situation, namely that of small spherically symmetric asymptotically flat initial data. In fact this appears to be the first time a theorem has been proved which guarantees the existence of non-static asymptotically flat spacetimes without singularities and containing normal matter in the sense indicated above. The theorem also shows that there is a striking contrast between the general relativistic collisionless gas and a superficially similar matter model, namely dust, which was studied in [4]. As discussed in Sect. 5, this could have significant consequences for the cosmic censorship hypothesis. In this paper the speed of light always has a fixed numerical value but it is also possible to replace this by a variable parameter in order to investigate the non-relativistic limit of the Vlasov-Einstein system. The nature of the local existence proof is such that it is feasible to control the equations uniformly in the parameter. It has been shown elsewhere [21] that under appropriate circumstances solutions of the Vlasov-Einstein system converge to solutions of the Vlasov-Poisson system as the speed of light tends to infinity.

The paper is organized as follows. Section 2 contains some basic information on the Vlasov-Einstein system in general and on the special case of solutions which are spherically symmetric and asymptotically flat. A reduced system of equations is introduced in the latter case, and it is shown that in order to solve the initial value problem for the Vlasov-Einstein system in that case it is enough to solve the reduced system. In Sect. 3 a local existence theorem is proved for the reduced system. At the same time a continuation criterion is obtained which says that if the maximum momentum in the support of the distribution function is finite on some time interval then the solution can be extended to a larger time interval. This may be compared with existing criteria in the Vlasov-Poisson [2] and Vlasov-Maxwell [13] cases. These results are applied in Sect. 4 to obtain global existence for small initial data together with information on the asymptotic behaviour of the solutions. An important element of the proof is the study of the rate at which geodesics spread on long time intervals. This is closely related to a technique used by Bardos and Degond [1] in their proof of global existence of solutions of the Vlasov-Poisson system for small initial data. However, the details of the argument in the present paper rely essentially on differential geometric ideas. It is shown that the global solutions constructed are singularity-free in the sense of the definition 
which is standard in general relativity, i.e. that they are timelike and null geodesically complete. In Sect. 5 the results of this paper are compared with those of Christodoulou [4] on spherically symmetric dust solutions, and possible implications for cosmic censorship are discussed.

\section{Preliminaries}

We consider the Vlasov-Einstein system for particles which all have the same mass. Units are chosen so that the gravitational constant, the speed of light and the mass of a particle all have the numerical value 1 . The sign conventions for geometrical objects are as in [15]. If $\left(M, g_{\alpha \beta}\right)$ is a given time-oriented spacetime then the mass shell $P$ is the submanifold of the tangent bundle $T M$ of $M$ consisting of vectors $p^{\alpha}$ which satisfy $g_{\alpha \beta} p^{\alpha} p^{\beta}=-1$ and are future pointing. The geodesics of the metric $g_{\alpha \beta}$ are the projections onto spacetime of the curves in $T M$ defined in local coordinates by

$$
\left.\begin{array}{l}
\frac{d x^{\alpha}}{d s}=p^{\alpha} \\
\frac{d p^{\alpha}}{d s}=-\Gamma_{\beta \gamma}^{\alpha} p^{\beta} p^{\gamma}
\end{array}\right\}
$$

where $\Gamma_{\beta \gamma}^{\alpha}$ are the Christoffel symbols. The solutions of (2.1) are the integral curves of the vector field (geodesic spray) $X=p^{\alpha} \frac{\partial}{\partial x^{\alpha}}-\Gamma_{\beta \gamma}^{\alpha} p^{\beta} p^{\gamma} \frac{\partial}{\partial p^{\alpha}} . X$ is tangent to $P$. The Vlasov equation for a non-negative real-valued function $f$ on $P$ is $X f=0$. Given a function $f: P \rightarrow \mathbb{R}$ define the particle current density and the energymomentum tensor by

$$
\begin{aligned}
N^{\alpha} & :=-\int f p^{\alpha}|g|^{1 / 2} / p_{0} d p^{1} d p^{2} d p^{3}, \\
T^{\alpha \beta} & :=-\int f p^{\alpha} p^{\beta}|g|^{1 / 2} / p_{0} d p^{1} d p^{2} d p^{3} .
\end{aligned}
$$

Here it is understood that $P$ has been coordinatized by $x^{\alpha}$ and $p^{a}$ (Latin indices run from 1 to 3 ) and the coordinates are such that $p^{0}>0$ is equivalent to $p^{\alpha}$ being future-pointing for vectors satisfying $g_{\alpha \beta} p^{\alpha} p^{\beta}=-1$. The determinant of the metric $g_{\alpha \beta}$ is denoted by $g$. If $f$ satisfies the Vlasov equation then the covariant divergence of each of these objects vanishes.

$$
\begin{aligned}
& \nabla_{\alpha} N^{\alpha}=0, \\
& \nabla_{\alpha} T^{\alpha \beta}=0 .
\end{aligned}
$$

These relations can most easily be proved by using normal coordinates [12]. However, at this point we encounter a type of problem which will occur several times in what follows. The argument using a transformation to normal coordinates only works if $f$ is $C^{1}$ and $g_{\alpha \beta}$ is $C^{3}$. However, the solutions which will be constructed are less differentiable than this. The problem can be overcome by approximating the given objects by smoother ones and passing to the limit as will be shown below. The Einstein equation is

$$
G_{\alpha \beta}=8 \pi T_{\alpha \beta},
$$

where $G_{\alpha \beta}$ is the Einstein tensor of $g_{\alpha \beta}$. 
In the following these equations will be studied in the case where coordinates can be found such that the metric takes the form

$$
d s^{2}=-e^{2 \mu(t, r)} d t^{2}+e^{2 \lambda(t, r)} d r^{2}+r^{2}\left(d \theta^{2}+\sin ^{2} \theta d \phi^{2}\right) .
$$

Here the coordinates $(t, r)$ range over $[0, T) \times[0, \infty)$ for some $T>0$. Such a metric is spherically symmetric, i.e. it admits an action of the group $S O(3)$ by isometries. It will be assumed that $\lambda(t, 0)=0$ for all $t$, which means that a regular centre exists, and that $\mu$ and $\lambda$ tend to zero as $r \rightarrow \infty$, which means that the metric is asymptotically flat. Let $z_{0}$ and $z_{1}$ be the vectors $e^{-\mu} \partial / \partial t$ and $e^{-\lambda} \partial / \partial r$ respectively and define

$$
\rho:=T_{\alpha \beta} z_{0}^{\alpha} z_{0}^{\beta}, \quad p:=T_{\alpha \beta} z_{1}^{\alpha} z_{1}^{\beta} .
$$

Then two of the Einstein equations take the form (where a prime denotes a partial derivative with respect to $r$ )

$$
\begin{aligned}
& e^{-2 \lambda}\left(2 r \lambda^{\prime}-1\right)+1=8 \pi r^{2} \rho, \\
& e^{-2 \lambda}\left(2 r \mu^{\prime}+1\right)-1=8 \pi r^{2} p .
\end{aligned}
$$

If an auxiliary quantity is defined by

$$
m(t, r):=4 \pi \int_{0}^{r} s^{2} \rho(t, s) d s
$$

then (2.9) can be integrated to give

$$
e^{-2 \lambda(t, r)}=1-2 m(t, r) / r .
$$

It is clear from this equation that $\lambda \geqq 0$. Adding (2.9) and (2.10) gives

$$
2 r e^{-2 \lambda}\left(\lambda^{\prime}+\mu^{\prime}\right)=8 \pi r^{2}(\rho+p) .
$$

Hence $\lambda^{\prime}+\mu^{\prime} \geqq 0$. Since it has been assumed that $\lambda+\mu \rightarrow 0$ as $r \rightarrow \infty$ this implies that $\mu \leqq-\lambda \leqq 0$.

While it is important to use polar coordinates for the Einstein equation it turns out to be more convenient to use Cartesian coordinates for the Vlasov equation in this problem. Define therefore $x^{1}:=r \sin \theta \cos \phi, x^{2}:=r \sin \theta \sin \phi$ and $x^{3}:=r \cos \theta$ and let $x^{0}=t$. Then in these coordinates the metric takes the form

$$
d s^{2}=-e^{2 \mu(t, r)} d t^{2}+\left[\delta_{a b}+\left(e^{2 \lambda(t, r)}-1\right) \delta_{a c} \delta_{b d} x^{c} x^{d} / r^{2}\right] d x^{a} d x^{b} .
$$

Let $\left(x^{\alpha}, p^{\alpha}\right)$ denote the coordinates on the tangent bundle naturally associated with $x^{\alpha}$. The mass shell can be coordinatized by $\left(x^{\alpha}, p^{a}\right)$ but there is a better choice for the present purposes. Define a frame on $\mathbb{R}^{3}$ by

$$
e_{i}^{a}:=\delta_{i}^{a}+\left(e^{-\lambda}-1\right) x^{a} \delta_{i b} x^{b} / r^{2} .
$$

Here $i$ is a frame index and $a$ a coordinate index. This frame is orthonormal with respect to the metric (2.13). A vector in $\mathbb{R}^{3}$ will be parametrized not by its coordinate components $p^{a}$ but by its components $v^{i}$ in the frame (2.14). Since $p^{a}=e_{i}^{a} v^{i}$ :

$$
v^{a}=p^{a}+\left(e^{\lambda}-1\right) x^{a} \delta_{b c} x^{b} p^{c} / r^{2}
$$


In the coordinates $\left(x^{\alpha}, v^{a}\right)$ the Vlasov equation has the explicit form

$$
\begin{aligned}
\frac{\partial f}{\partial t} & +e^{\mu}\left(1+|v|^{2}\right)^{-1 / 2}\left[v^{a}+\frac{e^{-\lambda}-1}{r} v_{r} x^{a}\right] \frac{\partial f}{\partial x^{a}} \\
& +\left[e^{\mu}\left(1+|v|^{2}\right)^{-1 / 2}\left(\frac{e^{-\lambda}-1}{r^{2}}\right)\left(|v|^{2} x^{a}-r v_{r} v^{a}\right)\right. \\
& \left.-\dot{\lambda} \frac{v_{r} x^{a}}{r}-e^{\mu-\lambda} \mu^{\prime} \frac{x^{a}}{r}\left(1+|v|^{2}\right)^{1 / 2}\right] \frac{\partial f}{\partial v^{a}}=0
\end{aligned}
$$

Here $v_{r}:=r^{-1} \delta_{a b} x^{a} v^{b},|v|^{2}:=\delta_{a b} v^{a} v^{b}$ and a dot denotes a partial derivative with respect to $t$. Equation (2.16) simplifies considerably if the function $f$ is spherically symmetric, i.e. if it is invariant under the action of $S O(3)$ on the mass shell induced by the action on spacetime corresponding to the spherical symmetry. In that case it can be shown that, locally in a neighbourhood of any point of the dense subset of $P$ where the group acts freely, the function $f$ can be written as a function of the three invariants $r, v_{r}$ and $|v|$. It follows that

$$
\left(r^{2} v^{a}-r v_{r} x^{a}\right) \frac{\partial f}{\partial x^{a}}=\left(|v|^{2} x^{a}-r v_{r} v^{a}\right) \frac{\partial f}{\partial v^{a}}
$$

so that $(2.16)$ is equivalent to the equation

$$
\frac{\partial f}{\partial t}+e^{\mu-\lambda}\left(1+|v|^{2}\right)^{-1 / 2} v^{a} \frac{\partial f}{\partial x^{a}}-r^{-1} x^{a}\left(\dot{\lambda} v_{r}+e^{\mu-\lambda}\left(1+|v|^{2}\right)^{1 / 2} \mu^{\prime}\right) \frac{\partial f}{\partial v^{a}}=0
$$

The only derivatives of the metric coefficients which occur here are $\dot{\lambda}$ and $\mu^{\prime}$. In the coordinates $\left(x^{\alpha}, p^{a}\right)$ the derivative $\lambda^{\prime}$ also occurs in the Vlasov equation, and this leads to problems in treating the equation directly in that form. The explicit expressions for $\rho$ and $p$ in these coordinates are

$$
\begin{aligned}
& \rho\left(x^{\alpha}\right)=\int f\left(x^{\alpha}, v^{a}\right)\left(1+|v|^{2}\right)^{1 / 2} d v^{1} d v^{2} d v^{3}, \\
& p\left(x^{\alpha}\right)=\int f\left(x^{\alpha}, v^{a}\right) v_{r}^{2}\left(1+|v|^{2}\right)^{-1 / 2} d v^{1} d v^{2} d v^{3} .
\end{aligned}
$$

The spherically symmetric Vlasov-Einstein system consists of (2.18) together with (2.9) and (2.10) (with $\rho$ and $p$ given by (2.19) and (2.20).) In fact (2.9) and (2.10) only constitute a subset of the Einstein equations but it will be shown below that the reduced system consisting of (2.9), (2.10) and (2.18) is in fact equivalent to the full Vlasov-Einstein system for a metric of the form (2.7) with the boundary conditions already assumed.

The question of the justification of (2.4) and (2.5) when the metric is not $C^{3}$ will now be answered. The kind of differentiability obtained in the existence theorems motivates the following definition.

Definition. A triple $(f, \lambda, \mu)$ is called regular if

(i) $f$ is non-negative, spherically symmetric and $C^{1}$ and $f(t)$ has compact support for each $t$, 
(ii) all partial derivatives of $\lambda$ and $\mu$ up to the second order except the second time derivatives exist and are continuous,

(iii) the quantities $\lambda, \lambda^{\prime}$ and $\mu^{\prime}$ vanish at $r=0$ for each $t$.

Suppose now that $(f, \lambda, \mu)$ is a regular triple and that $f$ satisfies the Vlasov equation with respect to the metric determined by $\mu$ and $\lambda$ via the formula (2.13). The assumption of regularity implies that the Christoffel symbols with respect to Cartesian coordinates are continuous. Thus it suffices, by continuity, to check that (2.4) and (2.5) hold at an arbitrary point with $r \neq 0$. Let $\lambda_{n}$ and $\mu_{n}$ be sequences of $C^{3}$ functions defined on an open neighbourhood of a given point with this property which converge uniformly to $\lambda$ and $\mu$ respectively together with all those partial derivatives whose existence is demanded in the definition of regularity. A sequence of metrics $g_{\alpha \beta}^{n}$ can then be defined by replacing $\lambda$ and $\mu$ in (2.13) by $\lambda_{n}$ and $\mu_{n}$ respectively. If $t_{0}$ denotes the value of $t$ at the given point, let $f_{n}$ be the solution of the Vlasov equation in the geometry determined by $g_{\alpha \beta}^{n}$ which agrees with $f$ on the hypersurface $t=t_{0}$. Each $f_{n}$ is $C^{1}$ and these functions converge uniformly together with all their first order partial derivatives to $f$. This is true because of the differentiability and convergence properties of the coefficients in the Vlasov equation which are rather easy to control provided the neighbourhood is chosen so that it contains no points with $r=0$. (If the neighbourhood did contain such points it would in fact still be true, as will be seen in the next section.) Since the analogues of (2.4) and (2.5) hold for each triple $\left(f_{n}, \lambda_{n}, \mu_{n}\right)$, and passing to the limit in these equations is justified, these equations hold under the sole hypothesis that $(f, \lambda, \mu)$ is regular.

It will now be shown that Eq. (2.4) leads to an a priori estimate for a solution of the Vlasov equation. It is equivalent to the equation

$$
\partial_{\alpha}\left(|g|^{1 / 2} N^{\alpha}\right)=0
$$

Here $g$ denotes the determinant of the metric $g_{\alpha \beta}$ in the given Cartesian coordinates. This shows that if $N^{0}$ has compact support on each slice of constant time (and this will always be the case in the situations considered in this paper) then the quantity $\int|g|^{1 / 2} N^{0} d x^{1} d x^{2} d x^{3}$ is time independent. This quantity is equal to

$$
M(\infty):=\iint e^{\lambda} f\left(t, x^{a}, v^{a}\right) d x^{a} d v^{a} .
$$

The equivalence of the reduced system with the full Vlasov-Einstein system will now be demonstrated.

Theorem 2.1. Let $(f, \lambda, \mu)$ be a regular solution of the reduced system defined by Eqs. (2.9), (2.10) and (2.18)-(2.20). Then all Einstein equations are satisfied.

Proof. Let $E_{\alpha \beta}=G_{\alpha \beta}-8 \pi T_{\alpha \beta}$. The aim is to use the equation $\nabla_{\alpha} E^{\alpha \beta}=0$ and in view of (2.5) this is equivalent to $\nabla_{\alpha} G^{\alpha \beta}=0$. The latter equation holds for any $C^{3}$ metric but once again the problem comes up that this amount of regularity is not available. One of the components of this equation has the following explicit form for a metric of the type (2.7):

$$
\partial_{t} G^{00}+\partial_{r} G^{01}+G^{00}(2 \dot{\mu}+\dot{\lambda})+G^{01}\left(3 \mu^{\prime}+\lambda^{\prime}+2 / r\right)+G^{11} e^{2(\lambda-\mu)} \dot{\lambda}=0 .
$$


Now approximate $\lambda$ and $\mu$ by $C^{3}$ functions as above. Then it is possible to pass to the limit in (2.23) and obtain that equation for the metric of interest. Combining this with (2.5) and using the fact that (2.9) and (2.10) are equivalent to the vanishing of $E^{00}$ and $E^{11}$ respectively we see that

$$
\partial_{r}\left(r^{2} E^{01}\right)+\left(3 \mu^{\prime}+\lambda^{\prime}\right)\left(r^{2} E^{01}\right)=0 .
$$

This implies that $E^{01}$ is zero. Now the fact that $f$ is $C^{1}$ implies that $T^{01}$ is $C^{1}$ and this means under the present circumstances that $G^{01}$ is $C^{1}$. If $G^{01}$ is calculated it turns out that this gives the statement that $\dot{\lambda}$ is $C^{1}$. In particular $\ddot{\lambda}$ exists and is continuous. Another component of the equation $\nabla_{\alpha} G^{\alpha \beta}=0$ reads

$$
\begin{aligned}
\partial_{t} G^{01} & +\partial_{r} G^{11}+G^{00} e^{2(\mu-\lambda)} \mu^{\prime}+G^{01}(3 \dot{\lambda}+\dot{\mu})+G^{11}\left(\mu^{\prime}+2 \lambda^{\prime}+2 / r\right) \\
& -r^{-1} e^{-2 \lambda} g_{A B} G^{A B}=0 .
\end{aligned}
$$

Here the upper case Latin indices take the values 2 and 3 and refer to the coordinates $\theta$ and $\phi$. The quantity $G^{A B}$ involves $\ddot{\lambda}$. If the approximating sequence is chosen so that $\ddot{\lambda}_{n}$ converges to $\ddot{\lambda}$ then it is possible to pass to the limit in (2.25). Combining with (2.5) gives $g_{A B} E^{A B}=0$. As a consequence of the symmetry $E^{A B}$ is proportional to $g^{A B}$ and so in fact $E^{A B}=0$. The components $E^{0 A}$ and $E^{1 A}$ also vanish because of the symmetry. Thus all Einstein equations are satisfied.

\section{Local Existence}

The aim of this section is to prove the existence of a local in time solution of (2.9), (2.10) and (2.18) corresponding to an initial datum $\stackrel{0}{f}$. For the convenience of the reader who is not interested in the derivation of the equations we present the full reduced system here in a notation which emphasizes its relationship to the VlasovPoisson system,

$$
\left\{\begin{array}{l}
\partial_{t} f+e^{\mu-\lambda}\left(1+|v|^{2}\right)^{-1 / 2} v \cdot \nabla_{x} f \\
-\left[r^{-2} x \cdot v \dot{\lambda}+r^{-1} e^{\mu-\lambda}\left(1+|v|^{2}\right)^{1 / 2} \mu^{\prime}\right] x \cdot \nabla_{v} f=0 \\
e^{-2 \lambda}\left(2 r \lambda^{\prime}-1\right)+1=8 \pi r^{2} \rho \\
e^{-2 \lambda}\left(2 r \mu^{\prime}+1\right)-1=8 \pi r^{2} p \\
\rho(t, x)=\int\left(1+|v|^{2}\right)^{1 / 2} f(t, x, v) d v \\
p(t, x)=\int r^{-2}(x \cdot v)^{2}\left(1+|v|^{2}\right)^{-1 / 2} f(t, x, v) d v
\end{array}\right.
$$

Here $r=|x|$ and $x \cdot v$ denotes the usual inner product of vectors in $\mathbb{R}^{3}$. The distribution function $f$ is assumed to be invariant under all rotations and hence $\rho$ and $p$ can be regarded as functions of $t$ and $r$. Spherically symmetric functions of $t$ and $x$ will be identified with functions of $t$ and $r$ whenever it is convenient.

Theorem 3.1. Let $\stackrel{0}{f} \geqq 0$ be a spherically symmetric function which is $C^{1}$ and has compact support. Suppose that

$$
\int_{|x|<r} \int\left(1+|v|^{2}\right)^{1 / 2} \stackrel{0}{f}(x, v) d v d x<r / 2
$$


for all $r>0$. Then there exists a unique regular spherically symmetric solution of (3.1) on an interval $[0, T)$ with $f(0, x, v)=\stackrel{0}{f}(x, v)$.

Note that the condition (3.2) cannot be weakened. For if it is not satisfied Eq. (2.9) has no solution for $t=0$. The proof of the theorem will be broken up into a number of lemmas and is based on an iteration of the following type. First define $\lambda_{0}(t, r)$ and $\mu_{0}(t, r)$ to be zero. If $\lambda_{n}$ and $\mu_{n}$ are given on a time interval $\left[0, T_{n}\right)$ then $f_{n}$ is defined to be the solution of the Vlasov equation with $\lambda$ and $\mu$ replaced by $\lambda_{n}$ and $\mu_{n}$ and initial datum $f$. This solution can be described in the following way. Let $\left(X_{n}, V_{n}\right)(s, t, x, v)$ be the solution of the characteristic system

$$
\begin{aligned}
& \frac{d X_{n}}{d s}=e^{\mu_{n}-\lambda_{n}}\left(1+\left|V_{n}\right|^{2}\right)^{-1 / 2} V_{n}, \\
& \frac{d V_{n}}{d s}=-\left(\left|X_{n}\right|^{-2} X_{n} \cdot V_{n} \dot{\lambda}_{n}+\left|X_{n}\right|^{-1} e^{\mu_{n}-\lambda_{n}}\left(1+\left|V_{n}\right|^{2}\right)^{1 / 2} \mu_{n}^{\prime}\right) X_{n}
\end{aligned}
$$

with $\left(X_{n}, V_{n}\right)(t, t, x, v)=(x, v)$. Then

$$
f_{n}(t, x, v)=\stackrel{0}{f}\left(\left(X_{n}, V_{n}\right)(0, t, x, v)\right) .
$$

If $f_{n}$ is given define

$$
\begin{aligned}
& \rho_{n}(t, x):=\int\left(1+|v|^{2}\right)^{1 / 2} f_{n}(t, x, v) d v, \\
& p_{n}(t, x):=\int r^{-2}(x \cdot v)^{2}\left(1+|v|^{2}\right)^{-1 / 2} f_{n}(t, x, v) d v .
\end{aligned}
$$

Define $m_{n}$ by replacing $\rho$ by $\rho_{n}$ in (2.11) and let

$$
T_{n+1}:=\sup \left\{t: 2 m_{n}(s, r) / r<1, \forall r>0,0 \leqq s \leqq t\right\} .
$$

To get $\lambda_{n+1}(t, r)$ solve (2.9) on the interval $\left[0, T_{n+1}\right)$ with $\rho$ replaced by $\rho_{n}$ using (2.11) and (2.12). Then determine $\mu_{n+1}(t, r)$ by solving (2.10) with $p$ replaced by $p_{n}$ with the boundary condition that $\mu_{n+1}(t, r) \rightarrow 0$ as $r \rightarrow \infty$ for each $t$.

Lemma 3.1. If $\stackrel{0}{f}$ is an initial datum satisfying the hypotheses of Theorem 3.1 the iterates $f_{n}, \lambda_{n}$ and $\mu_{n}$ exist and are regular on $\left[0, T_{n}\right)$ where $T_{n}>0$ for all $n$.

Proof. It will be supposed as inductive hypothesis that $\left(f_{n}, \lambda_{n}, \mu_{n}\right)$ is regular and that $\mu_{n} \leqq-\lambda_{n} \leqq 0$. Since $f_{n}$ is regular the quantities $\rho_{n}$ and $p_{n}$ are also $C^{1}$. It is not difficult to show that as a consequence $m_{n}(t, r) / r$ is $C^{2}$ at each fixed time and continuous in $t$ and $r$ (which implies in particular that $T_{n+1}>0$ ) and then that $\lambda_{n+1}$ and $\mu_{n+1}$ have all the properties required in the definition of regularity. The least trivial part of this is to show the continuity at $r=0$ of the quantity $m_{n}(t, r) / r^{3}$ which occurs in the expressions for the second derivatives of $\lambda_{n+1}$ and $\mu_{n+1}$. This can be done as follows:

$$
\begin{aligned}
\left|m_{n}(t, r) / r^{3}-(4 \pi / 3) \rho_{n}(t, 0)\right| & =4 \pi r^{-3}\left|\int_{0}^{r} s^{2}(\rho(t, s)-\rho(t, 0)) d s\right| \\
& \leqq(4 \pi / 3) \sup _{0 \leqq s \leqq r}|\rho(t, s)-\rho(t, 0)| \\
& \rightarrow 0 \text { as } r \rightarrow 0 .
\end{aligned}
$$


The fact that $\mu_{n+1} \leqq-\lambda_{n+1}<0$ can be seen just as in the case of the corresponding statement for $\lambda$ and $\mu$ proved in the last section. Consider next the problem of solving the characteristic system (3.3) with $n$ replaced by $n+1$. Its coefficients depend on $\lambda_{n+1}, \mu_{n+1}, \dot{\lambda}_{n+1}$ and $\mu_{n+1}^{\prime}$. It is easily seen that for $r \neq 0$ the coefficients in (3.3) and their first derivatives with respect to $x$ and $v$ are continuous. In fact all these quantities extend continuously to $r=0$ as a consequence of the regularity of $\lambda_{n+1}$ and $\mu_{n+1}$. Consider for example the expression

$$
\partial / \partial x^{a}\left(\mu_{n+1}^{\prime} x^{b} / r\right)=r^{-2} \mu_{n+1}^{\prime \prime} x^{a} x^{b}+r^{-3} \mu_{n+1}^{\prime}\left(r^{2} \delta^{a b}-x^{a} x^{b}\right) .
$$

Regularity implies that $\mu_{n+1}^{\prime}(r)=r \mu_{n+1}^{\prime \prime}(0)+o(r)$, and as a consequence this expression tends to $\mu_{n+1}^{\prime \prime}(0) \delta^{a b}$ as $r \rightarrow 0$. The proof of the continuous extendibility of other relevant quantities is similar but easier. It can be concluded that (3.3) has a unique $C^{1}$ solution on a short time interval [14]. Furthermore

$$
\begin{aligned}
&\left|\frac{d X_{n+1}}{d s}\right| \leqq 1 \\
&\left|\frac{d V_{n+1}}{d s}\right| \leqq\left(\left\|\dot{\lambda}_{n+1}(s)\right\|_{L^{\infty}}+\left\|\mu_{n+1}^{\prime}(s)\right\|_{L^{\infty}}\right)\left(1+\left|V_{n+1}\right|\right) .
\end{aligned}
$$

Thus the solution of the characteristic system can be extended to the whole of $\left[0, T_{n+1}\right)$. This means that we have recovered the statement of the inductive hypothesis with $n$ replaced by $n+1$. Since this statement is evident for $n=0$ it holds for all $n$.

Before going further we recall the following useful estimate (cf. [2]). Let $m(t, \infty)=\lim _{r \rightarrow \infty} m(t, r)$. Then for any $R>0$,

$$
m(t, r) / r^{2} \leqq(4 \pi / 3)\|\rho(t)\|_{L^{\infty}} R+m(t, \infty) R^{-2} .
$$

Putting $R=\left(3 m(t, \infty) / 2 \pi\|\rho(t)\|_{L^{\infty}}\right)^{1 / 3}$ in (3.10) gives

$$
m(t, r) / r^{2} \leqq\left(12 \pi^{2}\right)^{1 / 3}\|\rho(t)\|_{L^{\infty}}^{2 / 3}(m(t, \infty))^{1 / 3} .
$$

Lemma 3.2. If the hypotheses of Theorem 3.1 hold there exists some $T>0$ such that $T_{n} \geqq T$ for all $n$. The quantities $\lambda_{n}, \dot{\lambda}_{n}$ and $\mu_{n}^{\prime}$ are uniformly bounded in $n$ on the interval $[0, T)$.

Proof. For $t \in\left[0, T_{n}\right)$ define $P_{n}(t):=\sup \{|v|:(x, v) \in \operatorname{supp} f(t)\}$ and $Q_{n}(t):=$ $\left\|e^{2 \lambda_{n}(t)}\right\|_{L^{\infty}}$. The quantities $P_{n}(0)$ and $Q_{n}(0)$ are independent of $n$ for $n \geqq 1$; they will be denoted by $u_{0}$ and $q_{0}$ respectively. If a positive constant $C_{0}$ is given let $\left(z_{1}, z_{2}\right)$ be a solution of the system of integral equations

$$
\begin{aligned}
& z_{1}(t)=u_{0}+C_{0} \int_{0}^{t}(1+s)\left(1+z_{1}(s)\right)^{6} z_{2}^{2}(s) \exp \left[C_{0}(1+s)^{2}\left(1+z_{1}(s)\right)^{4} z_{2}(s)\right] d s, \\
& z_{2}(t)=q_{0}+C_{0} \int_{0}^{t}(1+s)\left(1+z_{1}(s)\right)^{5} z_{2}^{3}(s) \exp \left[C_{0}(1+s)^{2}\left(1+z_{1}(s)\right)^{4} z_{2}(s)\right] d s
\end{aligned}
$$

on a neighbourhood of zero. Let $T$ be any positive number such that a solution of these equations exists on $[0, T]$. It will be shown by induction that, provided the 
constant $C_{0}$ is chosen appropriately, for all $n$ the number $T_{n}$ is greater than or equal to $T$ and that on the interval $[0, T)$ the inequalities $P_{n}(t) \leqq z_{1}(t)$ and $Q_{n}(t) \leqq z_{2}(t)$ hold. In the course of the proof it will also be shown that $\lambda_{n}, \dot{\lambda}_{n}$ and $\mu_{n}^{\prime}$ can be bounded in terms of $P_{n}(t)$ and $Q_{n}(t)$ in a manner which is independent of $n$, and so this is enough to prove the lemma. Hence

The Vlasov equation implies that the quantity $\left\|f_{n}(t)\right\|_{L^{\infty}}$ is equal to $\|\stackrel{0}{f}\|_{L^{\infty}}$.

$$
\begin{aligned}
& \left\|\rho_{n}(t)\right\|_{L^{\infty}} \leqq(4 \pi / 3)\|\stackrel{0}{f}\|_{L^{\infty}}\left(1+P_{n}(t)\right)^{4}, \\
& \left\|p_{n}(t)\right\|_{L^{\infty}} \leqq(4 \pi / 3)\|\stackrel{0}{f}\|_{L^{\infty}}\left(1+P_{n}(t)\right)^{4} .
\end{aligned}
$$

Next note that the fact that $M(\infty)$ is independent of $t$ implies the inequality $\left\|f_{n}(t)\right\|_{L^{1}} \leqq \sqrt{q_{0}}\|\stackrel{0}{f}\|_{L^{1}}$. It follows that

$$
m_{n}(t, \infty) \leqq\left(1+P_{n}(t)\right)\|\stackrel{0}{f}\|_{L^{1}} \sqrt{q_{0}} .
$$

From now on $C$ will denote a constant only depending on the initial datum $\stackrel{0}{f}$ (and in particular not on $n$ ) whose value may change from line to line. Combining (3.11), (3.12) and (3.14) gives

$$
m_{n}(t, r) / r^{2} \leqq C\left(1+P_{n}(t)\right)^{3}
$$

Now

$$
\begin{aligned}
& \lambda_{n+1}^{\prime}=e^{2 \lambda_{n+1}}\left(-m_{n} / r^{2}+4 \pi r \rho_{n}\right), \\
& \mu_{n+1}^{\prime}=e^{2 \lambda_{n+1}}\left(m_{n} / r^{2}+4 \pi r p_{n}\right) .
\end{aligned}
$$

Applying (3.12), (3.13) and (3.15) gives

$$
\begin{aligned}
&\left\|\lambda_{n+1}^{\prime}(t)\right\|_{L^{\infty}} \leqq C(1+t)\left(1+P_{n}(t)\right)^{4} Q_{n+1}(t), \\
&\left\|\mu_{n+1}^{\prime}(t)\right\|_{L^{\infty}} \leqq C(1+t)\left(1+P_{n}(t)\right)^{4} Q_{n+1}(t) .
\end{aligned}
$$

Here the fact has been used that, because of (3.8), at time $t$ the supports of $\rho(t)$ and $p(t)$ are contained in the ball of radius $r_{0}+t$ about the origin, where $r_{0}$ is a bound for $|x|$ on the support of $\stackrel{0}{f}$. Estimating $\dot{\lambda}_{n+1}$ is more complicated.

$$
\dot{\lambda}_{n+1}(t, r)=4 \pi r^{-1} e^{2 \lambda_{n+1}} \int_{|x|<r} \int|x|^{2}(1+|v|)^{1 / 2} \dot{f}_{n}(t, x, v) d v d x .
$$

In handling this it is useful to notice that $r^{-1} \lambda_{n+1}^{\prime}$ and $r^{-1} \mu_{n+1}^{\prime}$ can be bounded by an expression of the form $C\left(1+P_{n}(t)\right)^{4} Q_{n+1}(t)$. Now substitute for $\dot{f}_{n}$ in (3.20) using the Vlasov equation. This introduces the quantities $\nabla_{x} f_{n}$ and $\nabla_{v} f_{n}$ and these must be got rid of by integration by parts in $x$ and $v$ respectively. Now

$$
\begin{aligned}
r^{-1} \iint_{|x|<r} e^{\mu_{n}-\lambda_{n}} v \cdot \nabla_{x} f_{n} d x d v= & r^{-1} \iint_{|x|=r}|x|^{-1}(x \cdot v) e^{\mu_{n}-\lambda_{n}} f_{n} d S d v \\
& -r^{-1} \iint_{|x|<r} e^{\mu_{n}-\lambda_{n}} v \cdot \nabla_{x}\left(\mu_{n}-\lambda_{n}\right) f_{n} d x d v \\
= & I_{1}+I_{2},
\end{aligned}
$$


say. Furth'ermore,

$$
\begin{aligned}
& r^{-1} \int_{|x|<r} \int\left(|x|^{-2}\left(1+|v|^{2}\right)^{1 / 2}(v \cdot x) \dot{\lambda}_{n}+|x|^{-1} e^{\mu_{n}-\lambda_{n}}\left(1+|v|^{2}\right) \mu^{\prime}\right) x \cdot \nabla_{v} f_{n} d v d x \\
& =-r^{-1} \int_{|x|<r} \int \dot{\lambda}_{n}\left[\left(1+|v|^{2}\right)^{1 / 2}+(v \cdot x / r)^{2}\left(1+|v|^{2}\right)^{-1 / 2}\right] f_{n} d v d x \\
& \quad-r^{-1} \int_{|x|<r} \int 2|x|^{-1} e^{\mu_{n}-\lambda_{n}}(x \cdot v) \mu_{n}^{\prime} f_{n} d v d x \\
& =I_{3}+I_{4},
\end{aligned}
$$

say. Using the estimates already obtained and taking account of the fact that $e^{\mu_{n}-\lambda_{n}} \leqq 1$ these integrals can be estimated as follows:

$$
\begin{aligned}
& \left|I_{1}\right| \leqq C(1+t)\left(1+\tilde{P}_{n}(t)\right)^{4}, \\
& \left|I_{2}\right| \leqq C\left(1+\tilde{P}_{n}(t)\right)^{5} Q_{n}(t) \\
& \left|I_{3}\right| \leqq C\left(1+\tilde{P}_{n}(t)\right)^{4} \int_{0}^{r} s\left|\dot{\lambda}_{n}(t, s)\right| \chi_{t} d s, \\
& \left|I_{4}\right| \leqq C\left(1+\tilde{P}_{n}(t)\right)^{5} Q_{n}(t) .
\end{aligned}
$$

Here $\tilde{P}_{n}(t)=\max \left\{P_{0}(t), \ldots, P_{n}(t)\right\}$ and $\chi_{t}$ denotes the characteristic function of the interval $\left[0, r_{0}+t\right]$. Putting all these together gives

$$
\left|\dot{\lambda}_{n+1}(t, r)\right| \leqq C(1+t)\left(1+\tilde{P}_{n}(t)\right)^{5} \tilde{Q}_{n+1}^{2}(t)+\left(1+\tilde{P}_{n}(t)\right)^{4} \tilde{Q}_{n+1}(t) \int_{0}^{r} s\left|\dot{\lambda}_{n}(t, s)\right| \chi_{t} d s,
$$

where $\tilde{Q}_{n}(t)=\max \left\{Q_{0}(t), \ldots, Q_{n}(t)\right\}$

Now define $\Lambda_{n}(t, r)=\max \left\{\left|\dot{\lambda}_{0}(t, r)\right|, \ldots,\left|\dot{\lambda}_{n}(t, r)\right|\right\}$. Then (3.21) implies an integral inequality for $\Lambda_{n+1}$. Applying Gronwall's lemma to this leads to

$$
\Lambda_{n+1}(t, r) \leqq C(1+t)\left(1+\tilde{P}_{n}(t)\right)^{5} \tilde{Q}_{n+1}^{2}(t) \exp \left[C(1+t)^{2}\left(1+\tilde{P}_{n}(t)\right)^{4} \tilde{Q}_{n+1}(t)\right]
$$

Putting (3.19) and (3.22) into (3.9) gives

$$
\begin{aligned}
P_{n+1}(t) \leqq u_{0} & +C \int_{0}^{t}(1+s)\left(1+\tilde{P}_{n}(s)\right)^{6} Q_{n+1}^{2}(s) \\
& \times \exp \left[C(1+s)^{2}\left(1+\tilde{P}_{n}(s)\right)^{4} Q_{n+1}(s)\right] d s .
\end{aligned}
$$

On the other hand

$$
\left|\frac{d}{d t}\left(e^{2 \lambda_{n+1}}\right)\right|=2\left|\dot{\lambda}_{n+1}(t, r)\right| e^{2 \lambda_{n+1}} \leqq 2 Q_{n+1}(t)\left\|\Lambda_{n+1}(t)\right\|_{L^{\infty}} .
$$

Hence by (3.22)

$$
\begin{aligned}
\tilde{Q}_{n+1}(t) \leqq q_{0} & +C \int_{0}^{t}(1+s)\left(1+\tilde{P}_{n}(s)\right)^{5} \tilde{Q}_{n+1}^{3}(s) \\
& \times \exp \left[C(1+s)^{2}\left(1+\tilde{P}_{n}(s)\right)^{4} \tilde{Q}_{n+1}(s)\right] d s
\end{aligned}
$$


We are now ready for the promised induction argument. Note first that $P_{0}(t)=u_{0}$, $Q_{0}(t)=1 \leqq q_{0}$ and $T_{0}=\infty$. Now suppose that for some $n$ we have $T_{n} \geqq T$, $P_{n}(t) \leqq z_{1}(t)$ and $Q_{n}(t) \leqq z_{2}(t)$. Then (3.23) and (3.25) hold on the interval [0, $\left.T_{n+1}\right)$. It is possible to replace $P_{n+1}$ and $\tilde{P}_{n}$ by $\tilde{P}_{n+1}$ in (3.23) and still get a valid inequality. Let $C_{0}$ be a value of the constant $C$ such that, for the given initial datum, these latter inequalities are valid. A comparison of the pair $\left(\widetilde{P}_{n+1}, \widetilde{Q}_{n+1}\right)$ with the pair $\left(z_{1}, z_{2}\right)$ shows that $P_{n+1}(t) \leqq z_{1}(t)$ and $Q_{n+1}(t) \leqq z_{2}(t)$ on $\left[0, T_{n+1}\right) \cap[0, T)$. Now $Q_{n+1}(t)$ tends to infinity as $t$ tends to $T_{n+1}$ while $z_{2}(t)$ is bounded on $[0, T)$. Hence $T_{n+1} \geqq T$, and the proof is complete.

Lemma 3.3. Under the hypotheses of Theorem 3.1 the quantities $\mu_{n}^{\prime \prime}$ and $\dot{\lambda}_{n}^{\prime}$ are uniformly bounded on the interval $[0, T)$ defined in Lemma 3.2.

Proof. First the expressions for the derivatives of interest will be examined. Only those terms which were not already estimated in the course of the proof of Lemma 3.2 will be written out explicitly,

$$
\begin{aligned}
& \mu_{n+1}^{\prime \prime}=4 \pi r e^{2 \lambda_{n+1}} p^{\prime} p_{n}^{\prime}+\ldots \\
& \dot{\lambda}_{n+1}^{\prime}=e^{2 \lambda_{n+1}}\left[-\dot{m}_{n} / r^{2}+4 \pi r \dot{\rho}_{n}\right]+\ldots .
\end{aligned}
$$

Differentiating (3.4) with respect to $t$ leads to an estimate of the form

$$
\left\|\dot{f}_{n}\right\|_{L^{\infty}} \leqq C\|\stackrel{0}{f}\|_{C^{1}}
$$

With this estimate it is straightforward to show that $\dot{\rho}_{n}$ and $\dot{m}_{n}(r) / r^{2}$ are bounded. The boundedness of $\dot{\lambda}_{n}^{\prime}$ follows. It only remains to take care of $p_{n}^{\prime}$. To do this multiply the Vlasov equation by $(x \cdot v) / r$ and integrate the resulting equation with respect to the variable $v$. The term involving $\nabla_{v} f_{n}$ can be estimated by partial integration while $\partial f_{n} / \partial t$ can be bounded using (3.28). There results a bound for

$$
\int r^{-1}(x \cdot v)\left(1+|v|^{2}\right)^{-1 / 2} v \cdot \nabla_{x} f_{n} d v
$$

Since $f_{n}$ is spherically symmetric equation (2.17) can be applied, and so this is enough to bound $r p_{n}^{\prime}$ and hence $\mu_{n+1}^{\prime \prime}$.

Lemma 3.4. Under the hypotheses of Theorem 3.1 the solutions $X_{n}, V_{n}$ of the characteristic system (3.3) and the quantities $\lambda_{n}, \mu_{n}, \mu_{n}^{\prime}, \dot{\lambda}_{n}$ converge uniformly on the interval $[0, T)$.

Proof. Note first that with the results of Lemma 3.2 in hand it is possible to obtain an estimate of the following form for the differences of the metric coefficients and their derivatives,

$$
\begin{array}{r}
\left\|\lambda_{n+1}(t)-\lambda_{n}(t)\right\|_{L^{\infty}}+\left\|\mu_{n+1}(t)-\mu_{n}(t)\right\|_{L^{\infty}}+\left\|\mu_{n+1}^{\prime}(t)-\mu_{n}^{\prime}(t)\right\|_{L^{\infty}} \\
\leqq C\left\|f_{n}(t)-f_{n-1}(t)\right\|_{L^{\infty}} .
\end{array}
$$

In order that this make sense it is necessary to impose the restriction $n \geqq 1$. Define $\alpha_{n}(t)$ to be equal to

$$
\begin{gathered}
\sup \left\{\left(\left|X_{n+1}-X_{n}\right|+\left|V_{n+1}-V_{n}\right|\right)(s, t, x, v):\right. \\
\left.0 \leqq s \leqq t,(x, v) \in \operatorname{supp} f_{n+1}(t) \cup \operatorname{supp} f_{n}(t)\right\}
\end{gathered}
$$


Then by (3.4) for each $n \geqq 1$,

$$
\left\|f_{n}(t)-f_{n-1}(t)\right\|_{L^{\infty}} \leqq\|\stackrel{0}{f}\|_{C^{1} \alpha_{n-1}}(t) .
$$

To estimate the differences of the derivatives $\dot{\lambda}_{n}$ the same kind of argument is necessary as was used to bound $\dot{\lambda}_{n}$ above, namely express $\dot{\lambda}_{n}$ in terms of $\dot{f}_{n-1}$, substitute for the latter quantity using the Vlasov equation and eliminate the resulting derivatives of $f_{n-1}$ by partial integration. There results for $n \geqq 2$ the estimate

$$
\left|\dot{\lambda}_{n+1}(t, r)-\dot{\lambda}_{n}(t, r)\right| \leqq C\left(\alpha_{n-1}(t)+\alpha_{n-2}(t)+\int_{0}^{r} s\left|\dot{\lambda}_{n}(t, s)-\dot{\lambda}_{n-1}(t, s)\right| \chi_{t} d s\right) .
$$

Let $\beta_{n}(t)=\alpha_{n}(t)+\alpha_{n-1}(t)$. It can now be proved by induction (starting with $\left.n=2\right)$ that

$$
\left|\dot{\lambda}_{n+1}(t, r)-\dot{\lambda}_{n}(t, r)\right| \leqq C_{1} \frac{C^{n-1} r^{2(n-1)}}{2^{n-1}(n-1) !}+\sum_{i=1}^{n-1}\left[\frac{C^{n-i} r^{2(n-i-1)}}{2^{n-i-1}(n-i-1) !}\right] \beta_{i}(t),
$$

where $C_{1}=\left\|\dot{\lambda}_{1}\right\|_{L^{\infty}}+\left\|\dot{\lambda}_{2}\right\|_{L^{\infty}}$. Next the differences of the characteristics must be estimated. Using the results of Lemmas 3.2 and 3.3 it can be shown that

$$
\begin{aligned}
& \left|\frac{d X_{n+1}}{d s}-\frac{d X_{n}}{d s}\right|+\left|\frac{d V_{n+1}}{d s}-\frac{d V_{n}}{d s}\right| \leqq C\left(\left|X_{n+1}-X_{n}\right|+\left|V_{n+1}-V_{n}\right|\right. \\
& \quad+\left\|\lambda_{n+1}(s)-\lambda_{n}(s)\right\|_{L^{\infty}}+\left\|\mu_{n+1}(s)-\mu_{n}(s)\right\|_{L^{\infty}} \\
& \left.\quad+\left\|\dot{\lambda}_{n+1}(s)-\dot{\lambda}_{n}(s)\right\|_{L^{\infty}}+\left\|\mu_{n+1}^{\prime}(s)-\mu_{n}^{\prime}(s)\right\|_{L^{\infty}}\right) .
\end{aligned}
$$

Integrating this equation and using (3.29) and (3.30) leads to the integral inequality

$$
\alpha_{n}(t) \leqq C \int_{0}^{t}\left(\alpha_{n}(s)+\alpha_{n-1}(s)+\left\|\dot{\lambda}_{n+1}(s)-\dot{\lambda}_{n}(s)\right\|_{L^{\infty}}\right) d s
$$

This leads to a similar inequality for $\beta_{n}$ and summing the latter from 2 to $N$ gives

$$
\sum_{n=2}^{N} \beta_{n}(t) \leqq C\left(1+\int_{0}^{t} \sum_{n=2}^{N}\left(\beta_{n}(s)+\left\|\dot{\lambda}_{n+1}(s)-\dot{\lambda}_{n}(s)\right\|_{L^{\infty}}\right) d s\right)
$$

The inequality (3.31) will now be substituted into (3.34). Note that the characteristics are only affected by the values of $\dot{\lambda}$ on the support of the distribution function so that for this purpose $r$ can be replaced in (3.31) by $r_{0}+T$. There then occurs a double sum which can be estimated as follows:

$$
\sum_{n=2}^{N} \sum_{i=1}^{n-1}\left[\frac{C^{n-i}\left(r_{0}+T\right)^{2(n-i-1)}}{2^{n-i-1}(n-i-1) !} \beta_{i}(s)\right] \leqq\left(\sum_{n=2}^{N}\left[\frac{C}{n !}\left(C\left(r_{0}+T\right)^{2} / 2\right)^{n}\right]\right)\left(\sum_{n=2}^{N} \beta_{n}(s)\right) \text {. }
$$

The first sum on the right-hand side of (3.35) converges as $N \rightarrow \infty$, and the other sum which arises when (3.31) is substituted into (3.34) also converges in that limit. Replacing these sums by their limits gives a linear integral inequality for $\sum_{n=2}^{N} \beta_{n}(t)$ and so by Gronwall's inequality $\sum_{n=2}^{N} \beta_{n}(t)$ is bounded independently of $N$ and $t$ on 
the interval $[0, T)$. It follows that $X_{n}$ and $V_{n}$ are uniform Cauchy sequences and hence converge uniformly. The inequalities (3.29) and (3.30) then give the remaining claims in the statement of the lemma.

Proof of Theorem 3.1. The uniform convergence of $X_{n}$ and $V_{n}$ implies that of $f_{n}$ because of (3.4). The limiting function satisfies the initial condition $f(0, x, v)=\stackrel{0}{f}(x, v)$. From the definition of $\rho_{n}$ and $p_{n}$ they too converge. This in turn implies the convergence of $m_{n} / r, m_{n} / r^{2}$ and $m_{n} / r^{3}$. It follows from these facts and Lemma 3.4 that the field equations (2.9) and (2.10) are satisfied by the limiting functions. Differentiating (3.4) with respect to $t$ shows that $\partial_{t} f_{n}$ converges uniformly to $\partial_{t} f$. It is then immediate that $\dot{\rho}_{n}, \dot{m}_{n} / r$ and $\dot{\lambda}_{n}^{\prime}$ converge uniformly. The convergence of $\mu_{n}^{\prime \prime}$ can be obtained by using the same kind of estimate previously used to show its boundedness. Now enough is known about the limiting functions $\lambda$ and $\mu$ to see that the Vlasov equation in the geometry they determine has a $C^{1}$ solution with initial datum $f$. It obviously agrees with the limit $f$ of the $f_{n}$. Thus the triple $(f, \lambda, \mu)$ satisfies the reduced system and has the correct initial datum. Since $f$ is $C^{1}$ the regularity of $(f, \lambda, \mu)$ can be checked by using the same arguments as in the proof of Lemma 3.1. Thus it only remains to show uniqueness. Suppose that $(\bar{f}, \bar{\lambda}, \bar{\mu})$ is also a regular solution of the reduced system on $[0, T)$ with initial datum $\stackrel{0}{f}$. Let

$\alpha(t):=\sup \{(|X-\bar{X}|+|V-\bar{V}|)(s, t, x, v): 0 \leqq s \leqq t,(x, v) \in \operatorname{supp} f(t) \cup \operatorname{supp} \bar{f}(t)\}$.

It is enough to show the uniqueness on $\left[0, T^{\prime}\right)$ for an arbitrary $T^{\prime}<T$. The regularity of both solutions implies the boundedness of many quantities on $\left[0, T^{\prime}\right)$. Using this $|d X / d s-d \bar{X} / d s|$ and $|d V / d s-d \bar{V} / d s|$ can be bounded by $\alpha(t)$ and differences of metric coefficients and their derivatives as in (3.32) above. The analogues of (3.29) and (3.30) also hold and show that all these differences, with the exception of that involving $\lambda$, can be bounded using $\alpha(t)$. By a procedure analogous to that used twice already in connection with $\dot{\lambda}$ an integral inequality can be obtained for the remaining difference, and applying Gronwall's lemma shows that this difference can also be bounded using $\alpha(t)$. It follows that

$$
\alpha(t) \leqq C \int_{0}^{t} \alpha(s) d s
$$

Gronwall's lemma now shows that $\alpha=0$, and the two solutions coincide.

Next a continuation criterion will be derived. For any solution $(f, \lambda, \mu)$ of the reduced system let $P(t):=\sup \{|v|:(x, v) \in \operatorname{supp} f(t)\}$ and $Q(t)=\left\|e^{2 \lambda}(t)\right\|_{L^{\infty}}$.

Theorem 3.2. Let $(f, \lambda, \mu)$ be a regular solution of the reduced system on an interval $[0, T)$ with compactly supported initial datum $\stackrel{0}{f}$. If $T<\infty$ and $[0, T)$ is the right maximal interval of existence then $P$ is unbounded.

Proof. We suppose that $P$ is bounded and show that the solution is extendible. Define

$$
j(t, x):=\int r^{-1}(x \cdot v) f(t, x, v) d v
$$


By Theorem 2.1 the Einstein equations which are not part of the reduced system are satisfied. One of these reads

$$
\dot{\lambda}=-4 \pi r e^{\mu+\lambda} j .
$$

If $P$ is bounded then $r j$ is bounded. Also $e^{\mu+\lambda} \leqq 1$. Hence $\dot{\lambda}$ is bounded. Integrating in $t$ shows that $\lambda$ and hence $Q$ is bounded. Consider now the question of what determines the time of existence obtained in Theorem 3.1. This is only limited by the size of the constant $C_{0}$ required in the proof of Lemma 3.2 and the size of $u_{0}$ and $q_{0}$. The size of $C_{0}$ is determined by $\|\stackrel{0}{f}\|_{L^{1}},\|\stackrel{0}{f}\|_{L^{\infty}}$ and $r_{0}$. Since $P$ and $Q$ are bounded on $[0, T)$ all the quantities which influence the size of the interval of existence obtained when giving $f\left(t_{0}\right)$ as initial datum at $t=t_{0}$ are also bounded. Hence a solution exists on $[t, t+\delta)$ for all $t \in[0, T)$ and some $\delta>0$ independent of $t$. The extendibility of the given solution then follows.

\section{Global Existence}

In this section global existence of solutions of the Vlasov-Einstein system will be proved for small spherically symmetric initial data. The essential ingredient of the proof is an examination of the long-time effects of geodesic deviation. Before coming to that, however, we need to establish some properties of spherically symmetric solutions of the Einstein equations where the energy-momentum tensor has certain decay properties.

Lemma 4.1. Consider a spherically symmetric asymptotically flat solution of the Einstein equations on a time interval $[0, T)$ with the property that the support of the restriction of $T^{\alpha \beta}$ to each hypersurface $t=$ const. is contained in the ball of radius $r_{0}+t$ about the centre and suppose that

$$
\left\|T^{\alpha \beta}(t)\right\|_{L^{\infty}}(1+t)^{2+\delta} \leqq K_{1}
$$

for some constant $K_{1}>0$ and some $\delta \in(0,1]$. Then

$$
\left\|\mu^{\prime}(t)\right\|_{L^{\infty}}+\|\dot{\lambda}(t)\|_{L^{\infty}}+\left\|\lambda^{\prime}(t)\right\|_{L^{\infty}}+\left\|r^{-1}\left(e^{-\lambda(t)}-1\right)\right\|_{L^{\infty}} \leqq C(1+t)^{-1-\delta},
$$

where the constant $C$ only depends on $K_{1}$ and the restriction of the solution to the initial hypersurface. Moreover $\lambda$ and $\mu$ can be uniformly bounded,

$$
\left\|\Gamma_{\beta \gamma}^{a}(t)\right\|_{L^{\infty}} \leqq C(1+t)^{-1-\delta}
$$

and the components of the Riemann curvature tensor satisfy

$$
\left\|R_{\beta \gamma \delta}^{\alpha}(t)\right\|_{L^{\infty}} \leqq C(1+t)^{-2-\delta} .
$$

Proof. In the vacuum region $e^{-2 \lambda(t, r)}=1-2 m(t, \infty) / r$. Differentiating with respect to $t$ and applying (3.38) gives $\frac{\partial}{\partial t}(m(t, \infty))=0$, which is the conservation of ADM mass in this situation. From (3.11) it follows that

$$
m(t, r) / r^{2} \leqq C(1+t)^{-\frac{4}{3}-\frac{2}{3} \delta} K_{1}^{\frac{2}{3}}
$$


for a constant $C$ only depending on the restriction of the solution to the initial hypersurface. From (3.38)

$$
\|\dot{\lambda}(t)\|_{L^{\infty}} \leqq C(1+t)^{-1-\delta} .
$$

Integrating in time shows that $\lambda$ can be bounded in terms of a constant multiple of its values at $t=0$. Next,

$$
\|r p(t)\|_{L^{\infty}} \leqq C\left\|T^{11}(t)\right\|_{L^{\infty}}(1+t) \leqq C(1+t)^{-1-\delta} K_{1} .
$$

Using the field equation for $\mu^{\prime}$ now shows that

$$
\left\|\mu^{\prime}(t)\right\|_{L^{\infty}} \leqq C(1+t)^{-1-\delta},
$$

where $C$ depends only on the restriction of the solution to the initial hypersurface and $K_{1}$. This implies a bound for $|\mu|$ on the whole interval. The field equation for $\lambda^{\prime}$ shows that it satisfies an estimate similar to (4.6) and (4.8). The corresponding estimate for $\left(e^{-\lambda}-1\right) / r$ is a consequence of (4.5). This proves (4.2) and if the Christoffel symbols are computed it is immediately clear that (4.3) is implied by (4.2). In order to obtain the estimate for the curvature the following additional estimates are needed.

$$
\begin{gathered}
\left|\frac{e^{-2 \lambda}-1}{r^{2}}\right|=\frac{2 m(t, r)}{r^{3}} \leqq \frac{8 \pi}{3}\|\rho(t)\|_{L^{\infty}} \leqq C(1+t)^{-2-\delta}, \\
\left|r^{-1} \lambda^{\prime}(t, r)\right| \leqq C\left(m(t, r) / r^{3}+\|\rho(t)\|_{L^{\infty}}\right) \leqq C(1+t)^{-2-\delta} .
\end{gathered}
$$

Now

$$
R_{a b c}^{0}=e^{-2 \mu} r^{-1} \dot{\lambda}\left(\delta_{a b} \delta_{c d}-\delta_{a c} \delta_{b d}\right)\left(x^{d} / r\right) .
$$

Combining this with (3.38) shows that

$$
\left\|R_{a b c}^{0}(t)\right\|_{L^{\infty}} \leqq C(1+t)^{-2-\delta} .
$$

Next consider $R_{b c d}^{a}$. This is a sum of terms, each of which is the product of a bounded quantity with either $r^{-2}\left(e^{-2 \lambda}-1\right)$ or $r^{-1} \lambda^{\prime}$. Hence, by (4.9) and (4.10),

$$
\left\|R_{b c d}^{a}(t)\right\|_{L^{\infty}} \leqq C(1+t)^{-2-\delta} .
$$

Noting that $R_{a 0 b}^{0}=R_{a b}-R_{a c b}^{c}$ and that the Ricci tensor $R_{a b}$ can be constructed pointwise from the energy-momentum tensor and the metric we find that

$$
\left\|R_{a 0 b}^{0}(t)\right\|_{L^{\infty}} \leqq C(1+t)^{-2-\delta} .
$$

This completes the proof of the lemma.

The following lemma, which is an easy consequence of Gronwall's inequality, will be required in the following.

Lemma 4.2. Consider the ordinary differential equation $\frac{d u}{d t}=F(t, u)$ for a $C^{1}$ function $F$ satisfying the inequality $|F(t, u)| \leqq \eta(1+t)^{-1-\delta}(1+|u|)$ for some constants $\delta>0$ and $\eta>0$. Then given any initial datum at $t=0$ the corresponding solution exists on the whole of $[0, \infty)$ and satisfies the inequality $|u(t)-u(0)| \leqq$ $\frac{\eta}{\delta} \exp \frac{\eta}{\delta}(1+|u(0)|)$ 
Consider now a timelike geodesic $\gamma$ passing through a point with coordinates $\left(t, x^{a}\right)$ and suppose that it intersects the initial hypersurface $t=0$. Let $\tau$ be the proper time measured along $\gamma$, starting at $t=0$. Let $\left\{e_{\sigma}^{\prime}\right\}$ be an orthonormal frame along $\gamma$ which is parallelly transported and which is such that $e_{0}^{\prime}$ is the tangent vector to $\gamma$. Let $e^{\prime \sigma}$ be the dual coframe. A Jacobi field along $\gamma$ is the derivative with respect to the parameter of a one-parameter family of geodesics in which $\gamma$ is embedded. If it is expressed as a linear combination $Z^{s} e_{s}^{\prime}$ of $e_{1}^{\prime}, e_{2}^{\prime}$ and $e_{3}^{\prime}$ then $Z^{s}$ satisfies the equation (see [15], p. 96)

$$
\frac{d^{2} Z^{s}}{d \tau^{2}}=\left(R_{\beta \gamma \delta}^{\alpha} e_{\alpha}^{\prime s} e_{0}^{\prime \beta} e_{t}^{\prime \gamma} e_{0}^{\prime \delta}\right) Z^{t} .
$$

The quantity which must be estimated is the Jacobian determinant of the mapping

$$
v^{a} \mapsto \tilde{X}^{a}\left(0, t, x^{a}, v^{a}\right)
$$

for fixed $t$ and $x^{a}$, where $\tilde{X}^{a}\left(s, t, x^{a}, v^{a}\right)$ is defined in terms of the characteristic system of (2.16) in the same way as $X^{a}\left(s, t, x^{a}, v^{a}\right)$ was defined in terms of that of (2.18). This mapping can be described in words as follows. First construct the unit timelike vector $p^{\alpha}$ at the point with coordinates $\left(t, x^{a}\right)$ corresponding to $v^{a}$. Then follow the geodesic $\gamma$ through $\left(t, x^{a}\right)$ with tangent vector $p^{\alpha}$ backwards until it meets the hypersurface $t=0$. The derivative of this mapping takes vectors tangent to the mass shell at $e_{0}^{\prime}$ to vectors tangent to the initial hypersurface $t=0$. Now a vector tangent to $P$ at $e_{0}^{\prime}$ can be identified with an element of the tangent space to $M$ at $\left(t, x^{a}\right)$ which is orthogonal to $e_{0}^{\prime}$. This can then be expressed as a linear combination $Y^{s} e_{s}^{\prime}$. The derivative of (4.16) can now conveniently be written as a composition of three linear mappings from $\mathbb{R}^{3}$ to itself. The first linear mapping is the one sending $v^{a}$ to $Y^{s}$ which has just been described. Let $L_{2}\left(Y^{s}\right)$ be the value at $t=0$ of the solution of (4.15) with the initial data $Z^{s}=0$ and $\frac{d Z^{s}}{d \tau}=Y^{s}$ at the point $\left(t, x^{a}\right)$. Finally, let $L_{3}\left(Z^{s}\right)$ be defined by the Cartesian components of the vector obtained by projecting the vector $Z^{s} e_{s}^{\prime}$ onto the tangent space to the initial hypersurface along $e_{0}^{\prime}$. Then the derivative of (4.16) at the point of interest is $L_{3} L_{2} L_{1}$. It may be objected that the differentiability requirements on a geometry needed in order to derive the relation between (4.15) and (4.16) are stronger than those satisfied by a general regular solution of the Vlasov-Einstein system. However, Eq. (4.15) is used in the following only in twice integrated form, and the latter can be justified by approximating the given geometry by smoother ones.

Lemma 4.3. Consider spherically symmetric solutions of the Vlasov-Einstein system on intervals $[0, T)$ satisfying the following conditions:

$$
\left.\begin{array}{l}
\|\stackrel{0}{f}\|_{L^{\infty}} \leqq \varepsilon, \\
u_{0} \leqq K_{2}, \\
r_{0} \leqq K_{3},
\end{array}\right\},
$$

where $r_{0}=\sup \left\{\left|x^{a}\right|: T^{\alpha \beta}\left(0, x^{a}\right) \neq 0\right\}$, and the inequality (4.1). Then if $\varepsilon$ and $K_{1}$ are sufficiently small there exists a constant $K_{4}$, depending only on $\varepsilon, K_{1}, K_{2}$ and $K_{3}$, such that

$$
\left\|T^{\alpha \beta}(t)\right\|_{L^{\infty}} \leqq K_{4}(1+t)^{-3} .
$$


Proof. First note that $\|\stackrel{0}{f}\|_{L^{1}}$ can be bounded by $(4 \pi / 3)^{2} K_{2}^{3} K_{3}^{3} \varepsilon$ so that for fixed $K_{2}$ and $K_{3}$ making $\varepsilon$ small forces $\|\stackrel{0}{f}\|_{L^{1}}$ to be small. Lemma 4.1 provides a lot of information on the decay of geometric quantities as $t \rightarrow \infty$. In particular the estimate (4.2) can be combined with Lemma 4.2 to show that if $K_{1}$ is small enough

$$
P(t) \leqq u_{0}+1 \text {. }
$$

The inequality (4.19) implies a uniform bound for the Cartesian components of $e_{0}^{\prime}$ if the tangent vector to the geodesic $\gamma$ is contained in the support of the distribution function. (Only such geodesics are of interest here.) The remaining frame vectors $e_{s}^{\prime}$ satisfy

$$
e_{s, \beta}^{\prime \alpha} e_{0}^{\prime \beta}+\Gamma_{\beta \gamma}^{\alpha} e_{s}^{\prime \beta} e_{0}^{\prime \gamma}=0 .
$$

The spatial components of (4.20) can be written as

$$
\frac{d}{d \tau}\left(e_{s}^{\prime a}\right)=-\Gamma_{\beta \gamma}^{a} e_{s}^{\prime \beta} e_{0}^{\prime \gamma}
$$

To profit from this it is necessary to have some information about the relation between proper time and coordinate time along $\gamma$. In fact

$$
\frac{d \tau}{d t}=e^{\mu}\left(1+|v|^{2}\right)^{-1 / 2} .
$$

Since $|\mu|$ has already been bounded (4.21) and (4.22) imply that

$$
\left|\frac{d}{d t}\left(e_{s}^{\prime a}\right)\right| \leqq C\left(\left|e_{s}^{\prime a}\right|+\left|e_{s}^{\prime 0}\right|\right)(1+t)^{-1-\delta} .
$$

On the other hand, since $e_{s}^{\prime}$ is a unit vector $\left|e_{s}^{\prime 0}\right| \leqq C\left|e_{s}^{\prime a}\right|$. It follows by Lemma 4.2 that (4.23) implies the global boundedness of $e_{s}^{\prime a}$ and hence of $e_{s}^{\prime 0}$.

Consider now once again a timelike geodesic $\gamma$ whose tangent vector is contained in the support of the distribution function. As a consequence of (4.22) there are positive constants $C_{1}$ and $C_{2}$ such that

$$
C_{1} t \leqq \tau \leqq C_{2} t
$$

along $\gamma$. We can assume without loss of generality that $C_{2} \geqq 1$ and then

$$
(1+t)^{-2-\delta} \leqq C_{2}^{2+\delta}(1+\tau)^{-2-\delta} .
$$

Thus if $K_{t}^{s}:=R_{\beta \gamma \delta}^{\alpha} e_{\alpha}^{s} e_{0}^{\beta} e_{t}^{\prime \gamma} e_{0}^{\prime \delta}$ an estimate of the form

$$
\left|K_{t}^{s}(\tau)\right| \leqq C(1+\tau)^{-2-\delta}
$$

holds along $\gamma$. Let $\tau_{0}$ be the value of $\tau$ at the point $\left(t, x^{a}\right)$ and let $Z^{s}(\tau)$ be the solution of (4.15) with $Z^{s}\left(\tau_{0}\right)=0$ and $\frac{d Z^{s}}{d \tau}\left(\tau_{0}\right)=Y^{s}$. Let

$$
E^{s}(\tau):=Z^{s}(\tau)-\left(\tau-\tau_{0}\right) Y^{s}
$$

Then by Taylor's theorem

$$
E^{s}(\tau)=\int_{\tau_{0}}^{\tau}(\tau-\sigma)\left(\sigma-\tau_{0}\right) K_{t}^{s}(\sigma) Y^{t} d \sigma+\int_{\tau_{0}}^{\tau}(\tau-\sigma) K_{t}^{s}(\sigma) E^{t}(\sigma) d \sigma .
$$


The first integral can be estimated as follows:

$$
\begin{aligned}
\left|\int_{\tau}^{\tau_{0}}(\tau-\sigma)\left(\sigma-\tau_{0}\right) K_{t}^{s}(\sigma) Y^{t} d \sigma\right| & \leqq\left(\tau_{0}-\tau\right)\left|Y^{t}\right| \int_{\tau}^{\tau_{0}} C \sigma(1+\sigma)^{-2-\delta} d \sigma \\
& \leqq C\left(\tau_{0}-\tau\right)\left|Y^{t}\right|
\end{aligned}
$$

since $\int_{0}^{\infty} \sigma(1+\sigma)^{-2-\delta} d \sigma<\infty$. Combining (4.28) and (4.29) and applying Gronwall's inequality gives

$$
\left|E^{s}(\tau)\right| \leqq C\left(\tau_{0}-\tau\right)\left|Y^{t}\right| \exp \int_{\tau}^{\tau_{0}} C(\sigma-\tau)(1+\sigma)^{-2-\delta} d \sigma .
$$

Hence

$$
\left|E^{s}(\tau)\right| \leqq C\left(\tau_{0}-\tau\right)\left|Y^{t}\right|
$$

In fact $K_{1}$ could be chosen so that $C \leqq \frac{1}{2}$ and then using (4.31) and the definition of $E^{s}$ shows that

$$
\left|\operatorname{det} L_{2}\right| \geqq C \tau_{0}^{3}
$$

The inequality (4.24) shows that $\tau_{0}$ can be replaced by $t$ in (4.32). The determinant of $L_{1}$ can be bounded below uniformly for all geodesics whose tangent vectors are contained in the support of the distribution function. The same is true of the determinant of $L_{3}$. Thus (4.32) can be converted into the statement that

$$
\left|\operatorname{det}\left(\frac{\partial \tilde{X}^{a}}{\partial v^{b}}\right)\left(0, t, x^{a}, \cdot\right)\right| \geqq C t^{3} .
$$

This has been shown to be valid for all $v^{a}$ in the support of the distribution function. If we knew that the mapping (4.16) was injective then we could change variables from $v^{a}$ to $X^{a}$ in the definition of the energy-momentum tensor. As a consequence of (4.33) and the boundedness of $P$ this would give an estimate of the form (4.18), completing the proof of the lemma. It will now be shown that for $K_{1}$ sufficiently small this mapping is indeed injective. Suppose that, on the contrary, there are two distinct geodesics $\gamma_{0}$ and $\gamma_{1}$ starting at the point with coordinates $\left(t, x^{a}\right)$ which meet the initial hypersurface at the same point. Let $v_{0}^{a}$ and $v_{1}^{a}$ be the coordinates of their initial tangent vectors on the mass shell. For $l \in[0,1]$ let $v_{l}^{a}=(1-l) v_{0}^{a}+l v_{1}^{a}$. Let $\gamma_{l}$ be the geodesic with initial tangent vector corresponding to $v_{l}^{a}$. Denote the spatial coordinates of the point of intersection of $\gamma_{l}$ with the initial hypersurface $t=0$ by $\xi^{a}(l)$. Then $\xi^{a}(0)=\xi^{a}(1)$ and $\xi^{a}(l)$ is a closed curve. It can be concluded from (4.33) that the tangent vector to the curve $\xi^{a}(l)$ can never vanish. For each $\gamma_{l}$ choose the frame $e_{\sigma}^{\prime}$ so that $e_{1}^{\prime}$ lies in the plane spanned by the vectors on the mass shell with coordinates $v_{1}^{a}$ and $v_{2}^{a}$. Then $Y^{2}(l)=Y^{3}(l)=0$ for all $l$. The constant $C$ in (4.30) can be made as small as desired by making $K_{1}$ small. Hence $E^{s}(0)$ can be made very small in comparison to $\tau_{0} Y^{s}$. This implies that the angle between $Z^{s}(0)$ and $Y^{s}$ (considered as elements of $\mathbb{R}^{3}$ ) can be made as small as we wish. In other words $Z^{2}(0)$ and $Z^{3}(0)$ can be made small in comparison with $Z^{1}(0)$, uniformly in $l$. On the other hand making the constant $C$ in (4.3) small (which can also be achieved by reducing the size of $K_{1}$ ) means that $e_{0}^{\prime}$ and $e_{1}^{\prime}$ are close to vectors in the plane 
spanned by $\partial / \partial t$ and $\left(v_{0}^{a}-v_{1}^{a}\right) \partial / \partial x^{a}$. This can be proved by applying Lemma 4.2 to the geodesic equation and to the equation of parallel transport (4.20). It can be concluded that the angle between the tangent vector to the curve $\xi^{a}(l)$ and $v_{0}^{a}-v_{1}^{a}$ is always small. Due to the fact that the former never vanishes this is inconsistent with the curve being closed. This contradiction shows that in fact (4.16) is injective, as required.

Theorem 4.1. Let a non-negative $C^{1}$ compactly supported spherically symmetric initial datum $f$ for the Vlasov-Einstein system be given which satisfies the inequalities (4.17) for some positive constants $\varepsilon, K_{2}$ and $K_{3}$. Then if $\varepsilon$ is small enough the corresponding solution exists globally in time. Moreover, for this solution

$$
\left\|T^{\alpha \beta}(t)\right\|_{L^{\infty}} \leqq C(1+t)^{-3},
$$

the metric coefficients $\lambda$ and $\mu$ are bounded and the estimates (4.2)-(4.4) hold with $\delta=1$.

Proof. Let $K_{1}$ and $\varepsilon$ be positive constants which are small enough so that the conclusions of Lemma 4.3 hold for some $\delta<1$. Let $T_{1}$ be a positive number satisfying

$$
K_{4}\left(1+T_{1}\right)^{\delta-1}<K_{1}
$$

The constant $C_{0}$ appearing in the integral equations used in the proof of Lemma 3.2 must be chosen in a way which depends on the initial data. If (4.17) is satisfied it can be made as small as desired by making $\varepsilon$ small while keeping $K_{2}$ and $K_{3}$ fixed. This has the effect that the solution of the integral equations exists on any prescribed time interval if $\varepsilon$ is sufficiently small. Moreover the solution can be made smaller than any given positive number on that interval by the same means. The argument used to prove Theorem 3.1 then shows that the solution of the Vlasov-Einstein system with initial datum $f$ will exist on any prescribed time interval provided that $\varepsilon$ is small enough. Now restrict $\varepsilon$ so that the assumptions of the theorem imply that the local solution corresponding to $f$ actually exists on the interval $\left[0, T_{1}\right)$ and that on that interval (4.1) is satisfied. Consider a fixed initial datum $f$ satisfying (4.17). Define

$$
T_{*}=\sup \left\{T:\left\|T^{\alpha \beta}(t)\right\|_{L^{\infty}} \leqq K_{1}(1+t)^{-2-\delta} \forall t \in[0, T)\right\} .
$$

Here $T_{*}=\infty$ is possible. In fact we will show that it is the only possibility. For suppose $T_{*}<\infty$. The assumptions already made ensure that $T_{*} \geqq T_{1}$. The definitions of $T_{1}$ and $T_{*}$ and Lemma 4.3 then show that the continuation criterion is satisfied on $\left[0, T_{*}\right)$ and that

$$
\left\|T^{\alpha \beta}\left(T_{*}\right)\right\|<K_{1}\left(1+T_{*}\right)^{-2-\delta} .
$$

This means that the solution can be extended to a time interval $\left[0, T_{2}\right)$ with $T_{2}>T_{*}$. Also $T_{2}$ can be chosen so that (4.1) is satisfied there. This contradicts the definition of $T_{*}$ and so in fact it must be the case that $T_{*}=\infty$. The inequality (4.1) holds on $[0, \infty)$ for some $\delta<1$. Applying Lemma 4.3 shows that an inequality of this form also holds for $\delta=1$. The remaining conclusions of the theorem then follow from Lemma 4.1 . 
This theorem can be strengthened by observing that if $(f, \lambda, \mu)$ is a solution of (3.1) with initial datum $f$ then $\left(f_{\alpha}, \lambda_{\alpha}, \mu_{\alpha}\right)$ is a solution with initial datum $\stackrel{0}{f}_{\alpha}$, where

$$
\begin{aligned}
f_{\alpha}(t, x, v) & =\alpha^{2} f(\alpha t, \alpha x, v), \\
\lambda_{\alpha}(t, r) & =\lambda(\alpha t, \alpha r), \\
\mu_{\alpha}(t, r) & =\mu(\alpha t, \alpha r), \\
f_{\alpha}(x, v) & =\alpha^{2} \stackrel{0}{f}(\alpha x, v) .
\end{aligned}
$$

Fix positive constants $K_{2}$ and $K_{3}$ and choose $\varepsilon$ small enough so that (4.17) implies the existence of a global solution. We claim that a global solution exists for any initial datum $f$ satisfying $\|\stackrel{0}{f}\|_{L^{\infty}} r_{0}^{2} \leqq \varepsilon K_{3}^{2}$. To see this, choose $\alpha$ so that $r_{0, \alpha}=K_{3}$. Then it follows that $\left\|f_{\alpha}\right\|_{L^{\infty}} \leqq \varepsilon$. Hence there is a global solution of (3.1) with initial datum $f_{\alpha}$. A global solution with initial datum $f$ can now be obtained by using the inverse of the above transformation.

Up to now we have only discussed solutions on intervals of the form $[0, T)$ but the whole situation is time symmetric and so Theorem 4.1 gives solutions where $t$ ranges over the whole real line. From the theorem it can be seen that all these solutions satisfy an inequality of the form

$$
\left\|T^{\alpha \beta}(t)\right\|_{L^{\infty}} \leqq C(1+|t|)^{-3} .
$$

It follows that $\lambda$ and $\mu$ are uniformly bounded and that decay estimates can be obtained for the components of the curvature tensor as $|t| \rightarrow \infty$. Decay estimates are also obtained for the quantities $\dot{\lambda}, \mu^{\prime}$ and $\left(e^{-\lambda}-1\right) / r$ which all fall off like $|t|^{-2}$. Using this information in the equation for timelike geodesics shows that the solutions of that equation exist globally in $t$. It then follows from (4.22) that the spacetime is timelike geodesically complete. Consider next null geodesics. If these are parametrized by $t$ then the resulting equation is very similar to that for timelike geodesics: it is merely necessary to replace the expression $\sqrt{1+|v|^{2}}$ by $|v|$ in some places. The relation between an affine parameter $\tau$ and $t$ is obtained by the same process from (4.22). Using the fact that the tangent vector to a null geodesic can never become zero at any point so that $|v|$ can never become zero for finite $t$ null geodesic completeness can be shown in just the same way as timelike geodesic completeness. These spacetimes are in fact also spacelike geodesically complete but since this is only of marginal interest the proof will be omitted.

\section{Comparison with Dust Solutions}

The Vlasov equation is linear, and so there is no problem with considering distributional solutions. It also makes sense to talk about distributional solutions of the Vlasov-Einstein system provided the integration in $v$ which is carried out produces an energy-momentum tensor which is defined pointwise and not only in a distributional sense. One kind of solution of this type is that where $f$ takes the form

$$
f\left(x^{\alpha}, p^{a}\right)=-u_{0}|g|^{-1 / 2} \rho\left(x^{\alpha}\right) \delta\left(p^{a}-u^{a}\left(x^{\alpha}\right)\right),
$$


where $\rho$ and $u^{a}\left(x^{\alpha}\right)$ are smooth functions on spacetime. In this case the energymomentum tensor takes the form $T^{\alpha \beta}=\rho u^{\alpha} u^{\beta}$. Such an energy-momentum tensor represents dust, i.e. a fluid without pressure. Conversely any solution of the Einstein equation with dust arises from a distributional solution of the VlasovEinstein system in this way. Thus we see that dust solutions in general relativity arise formally from the Vlasov-Einstein system by a singular limiting process. It was found by Yodzis, Seifert and Müller zum Hagen [25] that spherically symmetric solutions of the Einstein equations with dust as matter model can form naked singularities; they produced a finite-dimensional family of such solutions. A more general investigation of the development of naked singularities in spherically symmetric dust spacetimes was carried out by Christodoulou [4]. He considered solutions for which the matter is at rest at some initial time. He also assumed that the initial density was monotonically decreasing from the centre outwards which rules out singularities of the type found in [25]. It was shown that in a variety of circumstances where these assumptions are satisfied a singularity develops at the centre which is locally or even globally naked, thus violating strong or even weak cosmic censorship. For the discussion here we only require one detailed feature of these results, namely that a naked singularity can occur even if the initial data are arbitrarily small. Of course the kind of smallness assumption on the data which we made to get global existence for the Vlasov-Einstein system does not make sense for dust. What we wish to claim is that there is no reasonable definition of smallness for dust which will rule out globally naked singularities since Christodoulou's results show that these can occur even if the initial density is compactly supported and the $L^{\infty}$ norms of it and all its partial derivatives of all orders are smaller than any prescribed collection of positive constants.

The wider significance of this observation for the cosmic censorship hypothesis is as follows. Our intuition about the nature of gravitational collapse in general relativity is based to a large extent on the exact solution of Oppenheimer and Snyder [18] which is a spherically symmetric dust solution. Perturbing this within the class of spherically symmetric dust solutions leads to a qualitative change in the behaviour (formation of naked singularities). The results of this paper provide evidence that this may merely be due to the pathological nature of dust as a matter model. Moving to the Vlasov equation could restore the standard picture where strong cosmic censorship is valid. To confirm this idea will, however, require a generalisation of the results of the present work to the case where the data are no longer assumed to be small.

Acknowledgements. One of the authors (ADR) would like to thank D. Christodoulou and B. Schmidt for useful discussions.

\section{References}

1. Bardos, C., Degond, P.: Global existence for the Vlasov-Poisson equation in three space variables with small initial data. Ann. Inst. H. Poincaré (Analyse non linéaire) 2, 101-118 (1985)

2. Batt, J.: Global symmetric solutions of the initial value problem of stellar dynamics. J. Diff. Eq. 25, 342-364 (1977)

3. Choquet-Bruhat, Y.: Problème de Cauchy pour le système integro differentiel d'EinsteinLiouville. Ann. Inst. Fourier, Grenoble 21, 181-201 (1971) 
4. Christodoulou, D.: Violation of cosmic censorship in the gravitational collapse of a dust cloud. Commun. Math. Phys. 93, 171-195 (1984)

5. Christodoulou, D.: The problem of a self-gravitating scalar field. Commun. Math. Phys. 105, 337-361 (1986)

6. Christodoulou, D.: Global existence of generalized solutions of the spherically symmetric Einstein-scalar equations in the large. Commun. Math. Phys. 106, 587-621 (1986)

7. Christodoulou, D.: The structure and uniqueness of generalized solutions of the spherically symmetric Einstein-scalar equations. Commun. Math. Phys. 109, 591-611 (1987)

8. Christodoulou, D.: A mathematical theory of gravitational collapse. Commun. Math. Phys. 109, 613-647 (1987)

9. Christodoulou, D.: The formation of black holes and singularities in spherically symmetric gravitational collapse. Commun. Pure Appl. Math. 44, 339-373 (1991)

10. Christodoulou, D.: Bounded variation solutions of the spherically symmetric Einstein-scalar field equations. Preprint, Courant Institute, 1991

11. Christodoulou, D., Klainerman, S.: The global nonlinear stability of the Minskowski space. To appear in Ann. of Math.

12. Ehlers, J.: Survey of general relativity theory. In: Israel, W. (ed.) Relativity, Astrophysics and Cosmology. Dordrecht: Reidel 1973

13. Glassey, R., Strauss, W.: Singularity formation in a collisionless plasma could only occur at high velocities. Arch. Rat. Mech. Anal. 92, 59-90 (1986)

14. Hartman, P.: Ordinary differential equations. Boston: Birkhäuser 1982

15. Hawking, S.W., Ellis, G.F.R.: The large-scale structure of space-time. Cambridge: Cambridge University Press 1973

16. John, F.: Formation of singularities in elastic waves. Lecture notes in physics, 195, Berlin, Heidelberg, New York: Springer 194-210 (1984)

17. Lions, P.-L., Perthame, B.: Propagation of moments and regularity for the three dimensional Vlasov-Poisson system. Invent. Math. 105, 415-430 (1991)

18. Oppenheimer, J.R., Snyder, H.: On continued gravitational contraction. Phys. Rev. 56, 455-459 (1939)

19. Pfaffelmoser, K.: Global classical solutions of the Vlasov-Poisson system in three dimensions for general initial data. J. Diff. Eq. 95, 281-303 (1992)

20. Rein, G.: Generic global solutions of the relativistic Vlasov-Maxwell system of plasma physics. Commun. Math. Phys. 135, 41-78 (1991)

21. Rein, G., Rendall, A.D.: The Newtonian limit of the spherically symmetric Vlasov-Einsein system. Commun. Math. Phys. 150, 585-591 (1992)

22. Rendall, A.D.: On the choice of matter model in general relativity. Preprint, 1992

23. Schaeffer, J.: Global existence of smooth solutions of the Vlasov-Poisson system in three dimensions. Commun. Partial Diff. Eq. 16, 1313-1336 (1991)

24. Sideris, T.: Formation of singularities in three-dimensional compressible fluids. Commun. Math. Phys. 101, 475-485 (1985)

25. Yodzis, P., Seifert, H.-J., Müller zum Hagen, H.: On the occurrence of naked singularities in general relativity. Commun. Math. Phys. 34, 135-148 (1973)

Communicated by S.-T. Yau

\section{Note added in proof.}

26. Rein, G., Rendall, A.D.: Smooth static solutions of the spherically symmetric Vlasov-Einstein system. To appear in Ann. Inst. H. Poincaré (Physique Théorique) 
